\title{
Artificial Cell Research as a Field that Connects Chemical, Biological and Philosophical Questions
}

\author{
Anna Deplazes-Zemp*
}

\begin{abstract}
This review article discusses the interdisciplinary nature and implications of artificial cell research. It starts from two historical theories: Gánti's chemoton model and the autopoiesis theory by Maturana and Varela. They both explain the transition from chemical molecules to biological cells. These models exemplify two different ways in which disciplines of chemistry, biology and philosophy can profit from each other. In the chemoton model, conclusions from one disciplinary approach are relevant for the other disciplines. In contrast, the autopoiesis model itself (rather than its conclusions) is transferred from one discipline to the other. The article closes by underpinning the relevance of artificial cell research for philosophy with reference to the on-going philosophical debates on emergence, biological functions and biocentrism.
\end{abstract}

Keywords: Artificial cell research · Autopoiesis · Chemoton · Interdisciplinary research · Philosophy

With the ambition of producing a simple albeit living cell starting from non-living molecules, scientists move from the classical domain of chemistry to that of biology. On the one hand, this transition happens at the level of the research subject, which changes from non-living molecules to a living entity. On the other hand, there is a shift at the conceptual level concerning the research approach. Chemists traditionally study specific molecules and singular reactions. In contrast, biological research concepts, models and questions focus on the living entity and biologists are most interested in the function that chemical molecules and reactions fulfil in maintaining the life of such entities. This being said, it must be added that the emergence of disciplines such as molecular biology or biochemistry, have blurred the strict separation between chemistry and biology in practice. Nevertheless, an-

${ }^{\star}$ Correspondence: Dr. A. Deplazes-Zemp Institute of Biomedical Ethics and History of Medicine University of Zurich

Winterthurerstr. 30, $\mathrm{CH}-8006$ Zurich

E-mail: deplazes@ethik.uzh.ch alytically, the two material categories and research approaches can still be separated. Sometimes, studying molecules and studying living organisms go hand in hand, and research findings of one classical discipline are often highly relevant for the other. This does, however, not invalidate the analytical difference between the chemical and biological categories with respect to material, research approach and addressed questions. Chemical as well as biological results and models in the context of this shift raise interesting questions for philosophy. More specifically, they are relevant for the philosophy of science and bioethics. The first field reflects on different methods, concepts, models and findings of the sciences in question, and the latter deals with ethical implications related to the discussed scientific research.

In this short review article, I would like to discuss artificial cell research in the context of the three disciplines of chemistry, biology and philosophy. Questions about life have been the research subject not only of scientific disciplines but also philosophy, social sciences, humanities, arts and religions. ${ }^{[1]}$ It will not be possible to address the relationship between all of these approaches in detail within such a review article. I thus decided to address the relationship between chemistry, biology and philosophy in two historical models that are repeatedly being consulted and quoted by current artificial cell researchers. As models that describe and explain the transition from chemical molecules to biological cells, they directly relate the laboratory work of artificial researchers to the more general questions about life. After an introduction, I will compare the relation between chemistry and biology in the two models and the implications for philosophy that their authors are considering. Finally, I will give some examples of philosophically relevant concepts that profit from findings in artificial cell research. This article will not provide a complete history of artificial cell research nor will it cover all the interactions and influences that take place between the discussed disciplines. Rather, the aim of this article is to raise the reader's awareness and interest for the inter- and trans-disciplinary nature of artificial cell research, and to use this research field as an example to illustrate fruitful interactions and influences between the disciplines of chemistry, biology and philosophy.

\section{The Transition from Chemistry to Biology in Artificial Cell Research}

In this article, the phrase 'artificial cell research' is used for research approaches, which aim at producing minimal living cells starting from lipid vesicles. The different contributions to this special issue show that research on and with artificial cells can pursue different research objectives and address different research questions. It would be inaccurate to describe research on artificial cells as a field driven by the unique aim to produce living cells. It is not likely that this ambitious aim will be achieved in the near future; further, scientists focus on more specific and often more applied research questions. Nevertheless, at least some artificial cell scientists are interested in the very general - and cross-disciplinary - question about the conditions and basic principles of life as well as the 
origin of life. In order to be able to produce an artificial living cell, one needs to start from a model that outlines the conditions and criteria that a minimal cell needs to fulfil in order to be considered as 'alive'. The two historical models that I would like to introduce are being used by influential artificial cell researchers as a starting point to develop their model on how a living cell could be synthesised from non-living molecules. The chemoton theory by Tibor Gánti received, for instance, significant attention in the textbook on protocells edited by Steen Rasmussen and colleagues. ${ }^{[2]}$ The second example is the autopoiesis theory by Humberto Maturana and Francisco Varela, which has repetitively been consulted and discussed by Pier Luigi Luisi and his collaborators. ${ }^{[3]}$

\section{Chemoton Model}

The chemoton model was developed by Tibor Gánti, a chemical engineer from Hungary. He first published this model in his book 'The principle of life' in Hungarian in 1971; it was published in English in 1987.[4] In this book, Gánti develops the idea that life can be studied at different levels. He speaks of life criteria, which fall into the research field of biology, the principle of life concerning the laws behind these criteria that he describes in his chemical chemoton theory, and finally, an approach to study life as a philosophical category. ${ }^{[4]}$ Gánti explicitly discusses and separates research on life along the three disciplines examined in this article. His main focus clearly lies in chemistry, the first level, where he develops his chemoton model.
The chemoton model is an abstraction of the minimal living system; it describes the simplest version of a system that can be called alive. It is present in every living being and absent in non-living entities. Gánti starts from observations of simple unicellular organisms and states that all living cells contain the three subsystems: cytoplasm, membrane and genetic material. ${ }^{[4]}$ For the chemoton model, he reduces these components into the following three abstract subsystems (Fig. 1): first, the metabolic subsystem found in the cytoplasm. It forms a reaction network that, starting from a nutrient $\mathrm{X}$, produces all components to reproduce itself as well as the other two subsystems. Moreover, it releases the waste product Y. Second, there is the membrane subsystem, which is capable of autocatalytic growth, and third the information subsystem, a reaction system that can produce macromolecules by template polycondensation. Byproducts of this subsystem are used to form the membrane. Gánti explains that this third subsystem thereby is able to control and couple the other two subsystems stoichiometrically and to synchronise growth. ${ }^{[5]} \mathrm{He}$ understands it as the abstraction of the genetic material. Modern protocell scientists refer to this model as an early schematic model and honour the stoichiometric coupling of the functional elements. However, they also identify limitations of the model, for instance, that the chemoton model only considers the role of metabolism for growth but not for maintenance. ${ }^{[5 a]}$ Another criticism refers to the feature that the information subsystem does not modulate the metabolism, or that 'nutrient' $X$ must be of a practically un-

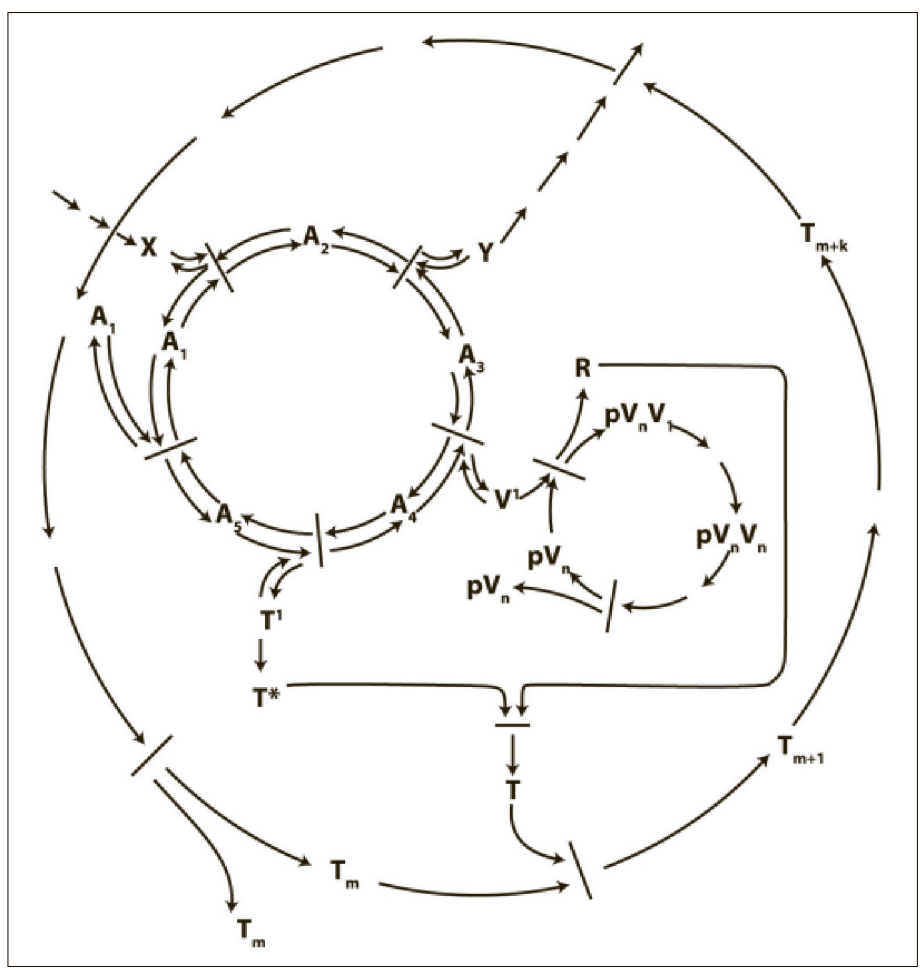

Fig. 1. Gánti's illustration of his chemoton model with its three stoichiometrically coupled subsystems. A: metabolic subsystem, T: membrane subsystem, V: information subsystem. Reproduced with permission from Oxford University Press. ${ }^{[4 b]}$ realistic size and complexity to serve as a source of all other components. ${ }^{[6]}$ What is important with regards to the relation between chemistry, biology and philosophy in artificial cell research, is the implication of this chemical model for research on life at the other levels.

As previously mentioned, Gánti's work also includes some reflections on the biological (rather than chemical) research approach to study life, which focuses on the criteria of life. The most inventive aspect of this part of his theory consists in his distinction between absolute and potential life criteria. The former must apply to every living organism, while the latter are not applicable to every individual organism but necessary for the "survival of the living world". ${ }^{[4]}$ He lists five absolute life criteria: (1) A living organism is an inherent unity with new qualitative properties compared to its parts; (2) Metabolism provides for transformation of external material and energy by the system; (3) Inherent stability means that the organisation of a living system remains stable in spite of changes in its environment; (4) An information-carrying subsystem contains the instructions for origin, function and development of the system; (5) Regulation and control of its processes is characteristic for living systems. The first of the three potential life criteria is (1) growth and reproduction, which are indispensable for the survival of a species but cannot be found in each organism. Gánti's distinction allows speaking of the controversially discussed feature of 'reproduction' as a life criterion without being forced to accept the implausible conclusion that sterile organisms cannot count as living entities. Capability of hereditary change and evolution is the second potential life criterion (2). Again, the possibility of potential criteria that concern the living world is an elegant solution for the problem that heredity and evolution does not occur at the level of individuals. These features can thus not count as criteria to describe a single organism as living. The final potential life criterion is (3) mortality. As Gánti writes "Non-living systems cannot die". ${ }^{[4, \mathrm{p} 79]}$ On the other hand, death is essential for the living world, because it allows for recycling of the organic material. The 'potential' nature of this criterion allows counting it as a criterion even though there are living systems, such as singular cells, whose life as an individual ceases by mitosis rather than by death. In this article, the question of how these biological criteria are connected to the chemical chemoton model is of particular interest. On the one hand, the chemoton, as the minimal living system, must satisfy the life criteria, which it clearly does as Gánti describes in detail.. ${ }^{[4]}$ On the other hand, Gánti writes that the chemoton theory describes the 
"principle of life" or the "laws uniting [the characteristics of life ]". .4, p74] Indeed, each of the absolute and potential life criteria depend in one way or another on the organisation described by the chemoton model. This organisation implies that independent and stable unities with new properties arise. In the chemoton model, this organisation obviously includes metabolism and the information system and allows for regulation and control. Moreover, it ensures the possibility of reproduction and heredity and thus evolution. Finally, the organisation can be abolished, which leads to death of the living system. In this sense, the chemical model is highly relevant for the understanding of the biological criteria.

What about the transition from chemistry/biology to philosophy? Although Gánti explains that he does not discuss life as a philosophical category, ${ }^{[4]}$ his final chapter and another essay titled 'Levels of life and death' do address certain philosophical implications of his chemoton theory and life criteria. ${ }^{[7]}$ The final chapter deals with the responsibility of the biologist, which Gánti explains against the background of his chemoton theory and life criteria. ${ }^{[4]}$ The irreversible functioning of the information subsystem, which in existing living organisms is implemented in the genetic programme, is the reason that direct or indirect effects on this system cause irreversible changes. Ganti writes: "It is this factor of irreversibility which makes biological manipulation incomparably more dangerous than manipulations performed on non-living systems. One can also produce catastrophes in the non-living world, but sooner or later every bad thing comes to an end. However, in the living world nothing really comes to an end, neither good nor bad. [...] Thus the effect of these events is in practice endless". ${ }^{[4,}$ p154] This inherent risk in "biological manipulations" leads to particular responsibilities for the biologist.

In the previously mentioned essay, Gánti advances his theory to animal life; he speaks of two different kinds of life: primary life that can be found already at the prokaryotic level and secondary life, which only occurs at the level of metazoa (multicellular animals). Interestingly, secondary life is organised by the same three-subsystem structure defined by the chemoton model. Analogous to the membrane subsystem, there is a subsystem governing the geometrical structure based on skin and bones. There is a subsystem involving a digestive tract, secretory organs and blood circulation that governs the metabolic processes in the whole animal. Finally, there is an information and control subsystem in the form of the nervous system.

The distinction between primary and secondary life is interesting for philosophical and ethical discussions about the be- ginning and end of human life. Whether brain death or cardiac death counts as criterion for human death is significant, for instance, with respect to organ donation. The removal of a vital organ from a brain-dead patient seems to be legally and morally less problematic if the body counts as 'dead'. At the beginning of life, the question of whether a human embryo should already count as a full human being is important, for example, for the ethical assessment of abortion. The biological definition of the beginning and end of life has turned out to be difficult, because, although a brain-dead patient or a human embryo do not live a typical human life, they doubtlessly show certain signs of life. Gánti's model may provide an explanation for this discrepancy. He argues that brain death should be the death criterion in the case of human life, because secondary life ends, even if certain primary life functions continue. Analogously, an oocyte or early embryo do have primary but not secondary life, which starts only with the functioning of the brain, the information and control subsystem at the secondary level. However, the ethically relevant question of why secondary life is the decisive criterion to decide whether an action is morally legitimate or not cannot be answered with reference to the scientific model alone. It demands some additional ethical argumentation, which would go beyond the scope of this article. The purpose of these comments is to show, how discussions pertaining to ethics and other branches of philosophy can profit from Gánti's chemical and biological theory.

\section{Autopoiesis Theory}

The second theory that has been influential concerning the material transition from chemistry to biology in the research field of artificial cells is the autopoiesis theory. Two Chilean biologists, Humberto Maturana and Francisco Varela, developed this theory. Their book 'Autopoiesis and Cognition, The Realization of the Living' was first published in Spanish in 1972; the English version followed in 1980.[8] The authors start from the question of whether there is a necessary and sufficient criterion for living organisms. For them, there is such a criterion, namely in the organisation of living systems, that allows them to be autonomous and self-producing unities. Maturana and Varela introduced the term 'autopoiesis' for self-production (ancient Greek: auto = self, poiesis = creation, production). Living organisms constantly maintain their own organisation; they are organised as a network of processes, which produces components that, in turn, participate in processes that realise and regenerate the network. This is to say that an autopoietic system is self-referential.
There is no difference between producer and product, the producer produces itself. This autopoietic system is captured within a determined boundary, which itself is of course produced by the system. The system thus constitutes an autonomous and clearly defined unity.

Maturana and Varela emphasise that their theory gets by without reference to a genome. As mentioned above, they claim that the notion of an autopoietic organisation is necessary and sufficient to characterise a living system. ${ }^{[8,9]}$ Some modern supporters of the autopoiesis theory put this claim for sufficiency into question. Pier Luigi Luisi and collaborators support their argument that autopoiesis is not sufficient to distinguish living from non-living systems with empirical evidence. In collaboration with Varela, Luisi started to develop models for experimental production of autopoietic chemical systems, ${ }^{[10]}$ which he and his research group then elaborated and applied towards the production of 'autopoietic vesicles' that are not alive. ${ }^{[11]}$ They described such a vesicle as: "a synthetic vesicle that absorbs a particular molecule from the medium, and by so doing, reproduces itself via an autocatalytic process".[3a, p170] What is the difference between such a vesicle and a simple living organism, for instance a bacterium? Bacteria do not take up their nutrients as a result of 'coincidental' chemical reactions. Their pre-existing metabolism only processes specific nutrients. This is a selective interaction with the environment. Although Maturana and Varela did not include this aspect into their theory of autopoiesis, it nevertheless has an important place in their work. They discuss this particular interaction that living things have with their environment as "cognition'. The choice of this term was unfortunate, because it is often associated with consciousness, which can certainly not be found in simple organisms such as bacteria. However, the meaning that Maturana and Varela give to this term has no connection to a nervous system or consciousness. Rather, they argue: "A cognitive system is a system whose organization defines a domain of interactions in which it can act with relevance to the maintenance of itself.' $[8, \mathrm{p} 13]$ It is thus the organisation of a living organism and therefore its autopoiesis that defines the cognitive domain of interaction. However, as the mentioned experiments of Luisi's group on non-living vesicles showed, not every type of autopoietic organisation leads to cognition. Together with the physicist and philosopher Michel Bitbol, Luisi argues that it is the metabolism, which implements the active and selective interaction with the environment that is characteristic of living systems. ${ }^{[12]}$ It can thus be concluded that autopoiesis alone is not sufficient to char- 
acterise life, but together with the condition of cognition the sufficiency criterion seems to be fulfilled.

The autopoiesis theory has been highly influential in social sciences and related branches of philosophy. De facto it has received much more attention in these fields than in the biological context where it was developed. Probably the best-known example is Niklas Luhmann's development of systems theory. He starts from the idea that social systems consist only of communication. He further applies the idea of autopoiesis to these systems. This means that social systems are constituted and differentiate from their environment by generating their own elements (communication) and maintain themselves strictly by internal operations. ${ }^{[13]}$ Luhmann writes: "Social systems use communication as their particular mode of autopoietic reproduction. Their elements are communications which are recursively produced and reproduced by a network of communications and which cannot exist outside of such a network." $[14$, p174] In contrast to the biological context, the material aspects of societies, such as human bodies, are thus part of the environment, which itself is not usually autopoietic.

The original authors of the autopoiesis theory had different opinions about the applicability of their theory to social systems. Maturana was in favour of the idea of autopoiesis for social systems. In contrast, Varela believed that talking about autopoiesis in social systems was an "abuse of language" because the notion of "boundary" as it was understood in autopoiesis did not apply to social systems. ${ }^{[3 a, p 176]}$

\section{Comparison and Discussion of the Chemoton and Autopoiesis Theories}

If one sets out to compare these two theories with regards to the transition from chemical molecules to biological cells, one is first struck by some marginal similarities in the circumstances of their origin. Both theories were developed independently from each other in countries outside the scientific centres in the early 1970s, and they were both first published in languages that were not influential in sciences. This background may, to some extent, explain why neither of the theories received much attention in the mainstream biochemical community. Luisi sees other reasons for this neglect in the fact that neither of the theories focused on nucleic acids as the main players, and that they provided systemic models rather than focusing on the importance of singular reactions. Nevertheless, Luisi explains that the idea to look at cellular life from a holistic, systemic point of view was part of a "Zeitgeist", which led to the simultaneous development of these similar theories in two entirely different environments. [3a, pp177-179]

A closer look at the content of the two theories reveals more detailed similarities underneath the holistic approach that both theories share. Not surprisingly, for both of them, the compartment or membrane plays a central role. This certainly is one reason why these are the theories used by artificial cell researchers. The metabolism, too, plays a key role in both theories. This is true, at least, if one compares the chemoton model with Luisi's version of the autopoiesis theory, which includes cognition (implemented in the metabolism) as a necessary element for life.

With respect to Gánti's third requirement - the information subsystem - the two theories differ. Although, as stated by Luisi, neither of the theories sets its primary focus on nucleic acids, Gánti's informational subsystem does refer to the genetic system as carrying out a function that is necessary for life. Several of Gánti's life criteria are conceptualised as depending on an informational system in the form of a hereditary programme. In contrast, Maturana and Varela emphasise that reference to a programme or information system is not requested for the characterisation of life. The development of the genetic system is, for proponents of the autopoiesis theory, one way to implement autopoiesis or maybe even an evolutionary consequence of autopoiesis. Reproduction and evolution depend on autopoietic units and are therefore secondary to the feature of autopoiesis. A kind of Lamarckian evolution already acts on very simple autopoietic entities. If self-reproductive autopoietic entities are able to adapt to their environment and reproduce in an adapted way, they will be more successful than non-adapted entities. In that sense, Maturana and Varela write: "In autopoietic systems evolution is a consequence of self-reproduction." $[8, \mathrm{p} 104]$ In the course of evolution as it happened on earth, the nucleic acid system became the key-feature of the self-reproduction of living entities. However, theoretically other models that involve the two key processes of evolution - namely self-reproduction and variation - are conceivable for autopoietic entities. ${ }^{[8,}$ pp102-106] From the perspective of Gánti's theory, one may question how reproduction of a membrane subsystem and a metabolism (as required for cognition) could practically be coordinated, if not by a type of information subsystem. However, even if an information system was requested for complex forms of life as we know them on earth, it could be argued that it is secondary to the feature of autopoiesis, since it 'evolved' as a consequence of autopoiesis.
Luisi identifies another divergence between the two theories in terms of their aim and purpose. ${ }^{[3 a,}$ pp178-179] Although Gánti himself did not experimentally work on artificial cells, he explicitly observed that his theory "involves the strategy of the synthesis of living systems"[3a, pp132ff] and can be consulted to explain the origin of life. [3a, pp140ff] The aim of Maturana and Varela, in contrast, was to develop a theory to describe what life is. In the context of this review article, this difference can be related to the different disciplinary backgrounds of the authors. The central aspect of Gánti's theory is driven by a chemical approach even though he also touches on biological questions with his life criteria. His main interest concerns the biochemical reactions that must take place in a minimal living system and how these reactions are coupled. He supports his model with various chemical equations. Maturana and Varela, on the other hand, are biologists interested in the phenomenon of life, in what distinguishes living from non-living things. Therefore, the phenomenological description was more central than the processes that implement this organisation. However, as discussed, both theories have far-reaching implications that go beyond their discipline of origin. Interestingly, the transfer from one discipline to the other(s) works differently in the two theories.

Gánti's 'chemical' chemoton model serves as an explanation for the biological criteria of life. These criteria are a continuation and further development of the chemical theory. They are derived from the chemoton model. Moreover, this model serves as an explanation for primary and secondary life, which explains certain philosophical and ethical implications as discussed above. In the case of autopoiesis, it is the model and not its conclusions that is transferred to the different disciplines. Luisi, as a chemist, tried to implement the biological model towards the chemical construction of artificial cells. This direct transfer is even more evident in the shift to social sciences and philosophy, where again, it is the model of organisation that is applied to social systems. So, these two theories give two different examples of how different academic disciplines can profit from each other's research. In the first example, the results and conclusions of one discipline are used to address research questions arising in other disciplines. In the second case, an explanatory model in one discipline is used to develop explanations to questions arising in other disciplines.

Finally, there is another 'external' difference that does not pertain to the content of the two theories. It concerns how they have been received within philosophy. In contrast to the autopoiesis theory, which 
received more attention in social sciences and philosophy than in biology, the implications of Gánti's model for philosophy, has hardly been discussed within this discipline. Thus, there would have been potential for an even more fruitful interaction between the disciplines with respect to the implications of the chemoton model. ${ }^{[15]}$

\section{Further Philosophical Questions Raised by Research on Artificial Cells}

As mentioned in the outset of this review article, the material transition from chemical molecules to biological cells according to the previously described models goes along with a conceptual shift in research questions and approaches. The focus on singular chemical reactions and players shifts to the effects that these reactions have for the new biological unity. This latter transition has various implications for philosophical research, as the third discipline discussed in this article. The artificial cell has first been discussed with respect to its chemical construction and its biological criteria; now I will address some of its philosophical characteristics. I already mentioned some implications and applications that resulted directly from the discussed scientific theories for philosophy and theoretical social sciences. In this section, I would like to briefly introduce three other concepts in philosophy that occur with the material transition from chemical molecules to a biological cell as a unity and the associated conceptual shift in research questions and approaches. I only refer to these discussions at the level of simple unicellular forms of life. Most of these questions are then further extended to what Gánti would call 'secondary life' in animals and humans, however, this raises many more issues that cannot be addressed here.

\section{Emergence}

The philosophical discussion, to which the transition from chemistry to biology probably contributes most directly, concerns the topic of emergence. ${ }^{[16]}$ How can we understand or explain the relation between the chemical basis of living systems and the novel properties that they have as living unities?[17] This difficulty not only arises at the transition from chemistry to biology. A classic example to discuss emergent properties is water, whose properties of being transparent and liquid cannot be explained with respect to the properties of singular $\mathrm{H}_{2} \mathrm{O}$ molecules. Marc Bedau defines emergent phenomena as phenomena that are, on the one hand, generated by underlying processes, and on the other hand, are autonomous from these very same processes. ${ }^{[18]}$ Emergent properties cannot be predicted from the non-relational properties of the components of the system. In that sense, Bedau suggests that the state of the system can only be derived from the state and dynamics of its parts by simulation. ${ }^{[18]}$ These 'emergentist' positions do not refer to any non-scientific metaphysical explanations for emergent phenomena or properties. They simply suggest that at a certain level of complexity, we need new types of scientific explanations, which - at least at the moment - cannot be derived from explanations at lower levels.

\section{Functions}

The observation that living entities have different subsystems and elements that contribute to maintaining this unity, is the starting point for another debate in the philosophy of biology. This is the discussion about biological functions. One can distinguish between roughly two approaches to define biological functions. The first approach addresses what Justin Garson called "etiological theories of functions". ${ }^{[19]}$ In a simplified description of these theories, it can be said that they try to give an answer to the question "why is this function there?", for instance, why do animals have eyes? ${ }^{[20]}$ Modern answers to this question refer to evolutionary explanations. Studies in evolution biology are thus more informative for this approach than research on artificial cells. The second approach deals with consequentialist theories of function in Garson's sense. These theories roughly understand functions as contributions to something, for instance to the survival of the living organisms. ${ }^{[21]}$ The discussion of functions in the context of artificial cells primarily supports consequentialist theories. If one understood functions exclusively etiologically with reference to evolution, one would need to conclude that at the level of artificial cells, there cannot be any biological functions, because they do not have any evolutionary history. ${ }^{[22]}$

\section{Interests}

The last point that I will briefly introduce concerns a much smaller debate than the two previous topics. Biocentrism is a position in environmental ethics, which argues that all living organisms are morally considerable. ${ }^{[23]}$ This means that to harm living organisms can morally only be justified, if one can give good reasons, why it is necessary to do so. But why should this be true specifically for living organisms? For the answer to this question, at least some authors directly refer to the previously mentioned discussion on functions. [24] External events can support or interfere with these functions and thereby with the maintenance of a living organism. This is morally relevant, because it means that something can be good or bad for the living system or in other words, it can support or go against the interest ${ }^{[25]}$ of the living organism. Another condition for having interests in this sense is related to the autopoiesis and chemoton theories. In order to be able to have interests, living organisms must be autonomous and able to maintain themselves, otherwise, their maintenance would not be in their own interest, but in that of their maintainer. The biocentric statement that having interest is the criterion for being morally considerable, because something can be against the interest for these entities, is certainly controversial. Nevertheless, it is a good example for how scientific conclusions can be informative for a philosophical subdiscipline.

\section{Conclusions}

In the emergence of disciplines such as synthetic biology, systems biology or various engineering disciplines (for instance molecular systems engineering) the boundaries between the classic disciplines are often blurred. One could speak of new transdisciplinary research fields. This development is explicitly not the focus of the review article at hand. Here, it was my aim to discuss how the three different approaches of chemistry, biology and philosophy can profit from each other (summarised in Fig. 2). It may be difficult to assign artificial cell research to one classic discipline, but it is clearly driven by different research questions, approaches and interpretations typical for the individual disciplines. I have tried to show how these approaches were combined and how they influenced each other. Two different sources for the fruitful nature of this interaction have been described. First, there is inspiration by an exchange of research results: The notion of the chemoton model as a minimal living system supported and explained Gánti's life criteria, and served as an explanation for the ethically relevant distinction between primary and secondary life. The empirical observations of emergent properties, the functions of different subsystems and the implications of these observations for the new biological unity was the starting point for studies in philosophy of biology and bioethics, such as those on emergence, functions and biocentrism. Second, the exchange of an explanatory model can be stimulating. The model of autopoietic self-organisation has been understood as a criterion for life by Maturana and Varela as an instruction for chemical synthesis of the cell by Luisi, and as a model for societal interactions by Luhmann. It can be hoped, that other emerging transdisciplinary fields will be open for an equally stimulating interaction between the involved disciplines. 


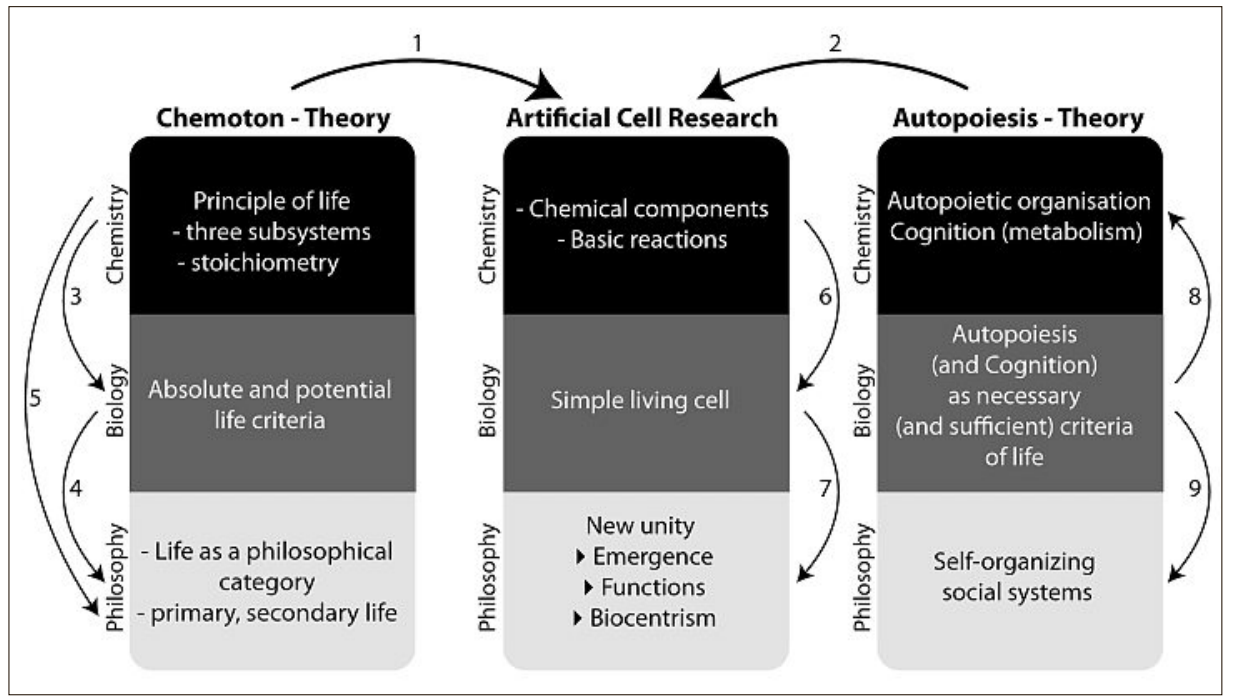

Fig. 2. Influences and inspiration between different disciplines in artificial cell research, the chemoton theory and the autopoiesis theory. The arrowheads illustrate how one discipline has influenced the others, as discussed in the text. Additional interactions are possible and likely. Artificial cell researchers have referred to the chemoton- and the autopoiesis theory as historical models that have influenced their research $(1,2)$. Gánti writes of research on life at three different levels. The chemoton model describes the principle of life, which explains absolute and potential life criteria (3). These life criteria support, for instance, particular responsibilities of scientists (4) and the principle of life constitutes the differences and parallels between primary and secondary life (5). In artificial cell research, the chemical composition of cells informs biological questions on the origin and minimal conditions of life (6). The production of a living cell goes along with the appearance of emergent properties, biological functions and - biocentrists argue - moral considerability (7). The autopoiesis model, which was developed in the biological context, has inspired artificial cell chemists (8) as well as social scientists and philosophers (9).

\section{Acknowledgements}

I would like to thank Sebastian Wäscher and Daniel Gregorowius for their valuable comments on an earlier version of the manuscript. This publication is supported by the Swiss National Science Foundation as part of the NCCR Molecular Systems Engineering.

\section{Received: March 22, 2016}

[1] a) A. Deplazes-Zemp, N. Biller-Andorno, EMBO Rep. 2012, 13, 959; b) A. DeplazesZemp, D. Gregorowius, N. Biller-Andorno, Nanoethics 2015, 9, 179 .

[2] S. Rasmussen, M. A. Bedau, L. Chen, D. Deamer, K. D. C., N. H. Packard, P. F. Stadler, 'Protocells, Bridging Nonliving and Living Matter', The MIT Press, Cambridge, MA London, 2009.

[3] a) P. L. Luisi, 'The Emergence of Life, From Chemical Origins to Synthetic Biology, Cambridge University Press, Cambridge, 2006; b) P. Stano, P. L. Luisi, Chem. Commun. 2010 , 46, 3639 .

[4] T. Gánti, in 'The Principles of Life', Eds. T. Gánti, E. Zsathmary, J. Griesmer, Oxford University Press, Oxford, 2003, p. 55.

[5] a) J. Griesmer, E. Szathmary, in 'Protocells, Bridging Nonliving and Living Matter', Eds. S. Rasmussen, M. A. Bedau, L. Chen, D. Deamer, K. D. C., N. H. Packard, P. F. Stadler, The MIT Press, Cambridge, MA London, 2009, p. 481; b) T. Gánti, 'The Principles of Life', Oxford University Press, Oxford, 2003.
[15] The article mentioned in ref. [7] is an exception, which does provide a thorough analysis of important philosophical implications of Gánti's model. It does, however, not address any ethical issues and thus does not take up the issues that Gánti raised himself.

[16] For a multifaceted introduction and discussion of this topic, which includes the scientific perspective, see M. A. Bedau, P. Humphreys, 'Emergence', The MIT Press, 2008.

[17] As this guiding question indicates, I discuss 'emergentism' here as an epistemological theory that captures how we explain and understand the world rather than an ontological theory that explains how the world is constituted. This reflects the approach to emergence taken by many contemporary authors, whereas historical theories often were understood in the latter sense. T. O'Connor, H. Y. Wong, in 'The Stanford Encyclopedia of Philosophy', Ed. E. N. Zalta, Stanford University, http://plato. stanford.edu/entries/properties-emergent/, Stanford, 2012, accessed January 2016.

[18] M. A. Bedau, Philosophical Perspectives: Mind, Causation, and World 1997, 11, 375.

[19] J. Garson, in 'A Companion to the Philosophy of Biology',Eds. S. Sarkar, A. Plutynsky, Blackwell Publishing, Blackwell Reference Online, http:// www.blackwellreference.com/subscriber/ tocnode.html?id=g9781405125727_chunk_ g978140512572730 2008, accessed January 2016.

[20] Besides Garson ${ }^{[19]}$, another informative review on the functions debate is provided by Mark Perlman: M. Perlman, The Monist 2004, 87, 3.

[21] Examples for representatives of the two theories are Robert Cummins as a supporter of a consequentialist theory: R. Cummins, in 'Philosophy of Biology, an Anthology', Eds. A. Rosenberg, R. Arp, Wiley-Blackwell, Malden, 2010, p. 164. and Ruth Milikan as a representative of etiological theories: $\mathrm{R}$. G. Milikan, 'Language, Thought, and Other Biological Categories', Bradford Books /MIT Press, 1984.

[22] Alternatively, I have suggested an adaptation of Gary Varner's etiological definition of biological functions to deal with synthetic organisms. G. E. Varner, The Southern Journal of Philosophy 1990, 28, 251; A. Deplazes-Zemp, Environ. Values 2012, 21, 63. This adaptation would allow for the characterisation of biological functions not exclusively by their evolutionary history but also by the fact that they can be adapted through evolution in the future.

[23] K. Goodpaster, J. Philosophy 1978, 75, 308.

[24] For instance, the biocentrist, Gary Varner, refers to his etiological definition of biological functions in order to explain (morally relevant) interests in living organism. ${ }^{[22]}$

[25] Importantly, by arguing that living organisms have interests, biocentrists are not claiming that simple living organisms have desires or intentions, but only that something can be good or bad for them. 